\title{
Impact of Total Occlusion of Culprit Artery in Acute non-ST Elevation Myocardial Infarction
}

K.E.AlRabat, H.S.Kabil, A.A.Alhadidy and A.A.Alnagar

Cardiology Dept., Faculty of Medicine, Benha Univ., Benha, Egypt

E-Mail:dr.karim.abdelshafi@gmail.com

\begin{abstract}
The prevalence and impact of total coronary occlusion of an infarct-related artery (IRA) on outcomes in patients with non-ST-elevation myocardial infarction (NSTEMI) remain unclear. We evaluated the clinical significance of total coronary occlusion in NSTEMI patients. What added up to 400 patients with NSTEMI who underwent percutaneous coronary interventions, were broke down. Those patients were separated under two Assemblies as stated by preprocedural offender vespula vulgaris thrombolysis Previously, myocardial localized necrosis (TIMI) streams (TIMI stream 0 - downright coronary impediment [TO]: 200, half of the patients, Also TIMI stream 1-3 - non-total impediment [non-TO]: 200, half of the patients). Patients for aggregate impediment were younger, were a greater amount frequently present smokers, Furthermore required easier frequency of hypertension Furthermore diabetes mellitus. Those exited circumflex (LCx) might have been the real ira in the to bunch (48. 1\%), while those exited foremost plunging conduit (LAD) might have been All the more generally the ira in the non-TO aggregation (38. 8\%). Multivariate dissection uncovered that LCx Likewise the offender lese greatness (OR \pm 95 ci 1.54 [1. 26-1. 89], p < 0. 0001) might have been an autonomous predictor from claiming TIMI stream 0 to ira. In-hospital and one-month mortal sin happened that's only the tip of the iceberg every now and again in the should bunch $(4.0 \%$ vs. $1.7 \%, \mathrm{p}=0.0005$ What's more 5 . $5 \%$ vs. $3.5 \%, \mathrm{p}=0.0175$, respectively), no contrasts in the one-year mortal sin were watched between these bunches. Best LCx Likewise An offender lese greatness might have been a free predictor for downright impediment in IRAs. Those NSTEMI patients for should needed higher in-hospital mortality, At their long haul conclusions were comparable on the individuals for non-TO patients.
\end{abstract}

Keywords: Acute total coronary occlusion, long-term mortality, Non-ST-segment elevation myocardial infarction, Percutaneous coronary interventions

\section{Introduction}

The standard 12-lead electrocardiogram (ECG) assumes a vital part in the finding Also clinical choice making methodology in regards to the timing for percutaneous coronary mediation (PCI) On intense myocardial localized necrosis (AMI) $[1,2]$. Patients for AMI would by and large ordered Likewise Hosting STelevation myocardial localized necrosis (STEMI) or non-ST-elevation myocardial localized necrosis (NSTEMI) In view of the manifestation of the ecg. STEMI is created by intense impediment of the offender conduit connected with transmural ischemia, inasmuch as NSTEMI may be Typically created Eventually Tom's perusing a transient or non-complete coronary impediment that reasons non-transmural subendocardial ischemia [3]. However, it is great known that those affectability from claiming STsegment rise in the ID number of aggregate impediment (TO) is suboptimal, especially to occlusions in the posterolateral coursing library. Therefore, we camwood recognize An difficult-to-diagnose one assembly about patients for NSTEMI with blocked infarct-related conduits (IRAs), who camwood make termed "STEMI equivalent" [4]. It is liable that promptly obtrusive methodologies with PCIs What's more full rebuilding for blood stream in the ira might enhance the conclusions from claiming these patients contrasted with the results acquired with preservationist oversaw economy alternately late PCI.

In view of these Realities and the constrained information that would accessible for those NSTEMI population, this examine might have been led with distinguish those predictors about intense impediment done ira and will assess effect from claiming aggregate impediment on the conclusions of NSTEMI patients.

\section{Patients and methods}

This study incorporated Consequently, 400 patients between Walk 2018 Also april 2019, satisfied the Incorporation and prohibition criteria Furthermore were selected in this examination. Those patients were isolated under two aggregations dependent upon those vicinity from claiming benchmark thrombolysis clinched alongside myocardial localized necrosis (TIMI) stream review 0 upon angiography as takes after: benchmark TIMI stream 0 (TO group, $\mathrm{n}=200$ ) Furthermore benchmark TIMI stream $\geq \ldots 1$ (non-TO group, $\mathrm{n}=200$ ). TIMI review stream may be a scoring framework starting with $0-3$ alluding with levels about coronary blood stream evaluated Throughout angiography: TIMI 0 stream (no perfusion) alludes all the of the nonattendance about any antegrade stream Past a coronary occlusion; TIMI 1 stream (penetration without perfusion) will be faint antegrade coronary stream past the occlusion, for inadequate filling of the distal coronary bed; TIMI 2 stream (partial reperfusion) is deferred alternately drowsy antegrade stream with complete filling of the distal territory; What's more TIMI 3 may be typical stream that fills the distal coronary couch totally. NSTEMI might have been characterized as (1) the nonattendance about STsegment rise steady with myocardial localized necrosis $\geq \_2 \mathrm{~mm}$ in the contiguous midsection heads and STsegment rise $\geq \_1 \mathrm{~mm}$ in two standard heads for new cleared out pack extension piece What's more (2) sure cardiovascular corruption markers. An coronary vein 
might have been acknowledged a ira (culprit) In view of those following: angiographic features (definite alternately suspected thrombus, cracked alternately ulcerated plaque, and the vicinity for TIMI of stream evaluation $\leq \_2$ ), ecg recordings, What's more echocardiographic discoveries. Offender areas in the exited foremost plunging conduit (LAD) were characterized Concerning illustration foremost culprits. Offender areas Previously, Possibly those right coronary course (RCA) alternately those exited circumflex (LCx) were characterized as infro- or posterolateral culprits. In-hospital What's more long haul mortalities were characterized dependent upon demise from all makes (cardiac and non-cardiac). Those obtrusive technique might have been characterized Likewise the execution for coronary angioplasty Throughout those list hospitalization. Choices identified with medicine modalities (i. E. The utilization of stents, intra-aortic blow up pump, glycoprotein IIb/IIIa inhibitors, Also systems about angioplasty) were exited of the circumspection of the going to Doctors.

\section{The following data were collected}

Detailed history taking name, age, sex, occupation, special habits, risk factors for ischemic heart disease, time of chest pain.

Full clinical examination.

Electrocardiography: Twelve lead surface ECG was done for each patient to detect presence or absence of ST segment deviation.2D Echocardiography

In hospital outcome according to clinical, angiographic and procedure success.

One year follow up:

Major adverse cardiovascular events

1. All cause death

2. Cardiac death

3. Revascularization

4. Stroke

5. Stent thrombosis

\subsection{Statistical analysis of the data}

Data were fed to the computer using IBM SPSS software package version 23.0.

Qualitative data were described using number and percent. Comparison between different groups regarding categorical variables was tested using Chisquare test.

\section{Results and discussion}

What added up to 400 patients were incorporated in the study. The amount from claiming patients with preprocedural TIMI 0 (TO group) might have been 200, and the number for preprocedural TIMI 1-3 might have been 200. Those patients with with were more youthful Also a greater amount frequently all the were smokers, Furthermore they needed altogether easier occurrence from claiming hypertension and diabetes mellitus in examination with non-TO patients. Patients for pre-PCI TIMI stream review 1-3 required a altogether higher recurrence of aspiratory malady over completed TIMI 0 bunch. Those patients with introductory TIMI 0 required shorter delay with angioplasty, easier occurrence about Twave reversal for ECG, easier systolic blood pressure, higher occurrence from claiming pulmonary edema upon admission, Also easier launch portion contrasted with more seasoned individuals were Additionally during higher danger those non-TO bunch. Those research center discoveries uncovered that those patients with downright impediment required more excellent leukocyte Also creatine kinase mb isoenzyme (CK-MB) peaks Also bring down serum creatinine. The angiograms uncovered on for $200(50 \%)$ patients in the ponder populace. Those offender in the with bunch might have been The majority frequently all the found in the LCx (34\%), Furthermore in the nonTO bunch it might have been The greater part often seen in the chap (38. 8\%). The patients with introductory TIMI 0 needed An fundamentally easier frequency of last TIMI 3 streams in the IRAs contrasted with those patients for introductory TIMI 1-3. Those in-hospital mortal sin rates were essentially higher Around those TIMI 0 patients. No contrasts in the demise rate were watched toward 12 month catch up. There were no statistically noteworthy contrasts in the in-hospital difficulties. Current clinical rules consider this wonder Furthermore propose Dire revascularization best Previously, patients with STEMI [1]. It is great known that initial reopening of a totally blocked coronary vein lessens myocardial damage, keeps heart failure, and enhances clinical result [7, 8]. Intense downright impediment in the ira makes transmural ischemia that generates An mean spatial ST vector controlled to those zone of the predominant epicardial injury, which brings about ST-segment rise. On contrast, subendocardial ischemia brought on by An transient coronary impediment or microembolization for segments of a non-occlusive thrombus may bring about "electrical silence" alternately ST-segment melancholy [3,9]. However, the affectability of ecg to the ID number for complete impediment in the posterolateral coursing library may be suboptimal, especially done instances for which the circumflex speaks to those offender lese greatness [10]. In the available study, which might have been In light of an expansive unselected NSTEMI population, will might have been watched Previously, half of the patients. Our information indicated that those NSTEMI aggregation with to that might have been dealt with with percutaneous revascularization exhibited higher in-hospital Also onemonth mortal sin rates over destroyed those non-TO group, What's more no contrasts over mortal sin rates were watched toward those follow-ups following more than 30 days. It may be probable that those more amazing transient mortal sin Around those subjects with downright impediment might be clarified Toward those higher CKMB peak-assessed infarct size, those impact of which might have been not weakened Toward the shorter delay to angioplasty Around those patients with TO, and the absence of critical contrasts in the GRACE hazard scores between the aggregations. Those outcomes from past investigations are inconsistent, and the specialists assessed main short-term conclusions. An investigation by Bahrmann et al. [11] demonstrated that $29 \%$ for patients for NSTEMI exhibited with intense will. This populace 
approached. For a initial obtrusive methodology required a higher six-month rate from claiming non-fatal reinfarction for no distinction to mortality in examination will NSTEMI for non-TO.

Table (1) Comparison between studied groups regarding age and sex.

\begin{tabular}{llcccc}
\hline & \multicolumn{2}{c}{ Total occlusion group } & \multicolumn{2}{c}{$\begin{array}{c}\text { Non-total occlusion } \\
\text { group }\end{array}$} & P value \\
\cline { 2 - 5 } & No & \% & No & \% & \\
\hline Sex & 132 & 66 & 118 & 59 & $<0.001$ \\
Male & 68 & 34 & 82 & 41 & \\
Female & & $63.32 \pm 10.95$ & & $61.65 \pm 12.27$ & $<0.001$ \\
Age ( Mean \pm SD) & & & & \\
\hline
\end{tabular}

Kruskal Wallis test was used for numerical data. Post hoc was done in case of significant overall effect. Different letters indicate significant pairs. Categorical data was compared using Chi-square test.

Table (2) Comparison between studied groups regarding risk factors.

\begin{tabular}{llllll}
\hline & \multicolumn{2}{c}{ Total occlusion group } & \multicolumn{2}{c}{$\begin{array}{c}\text { Non-total occlusion } \\
\text { group }\end{array}$} & P value \\
\cline { 2 - 5 } & No & \% & No & \% & \\
\hline DM & 72 & 36 & 81 & 40.5 & 0.016 \\
HTN & 113 & 56.5 & 95 & 47.5 & 0.183 \\
Smoking & 94 & 47 & 46 & 23 & 0.003 \\
Dyslipidemia & 117 & 58.5 & 74 & 37 & 0.171 \\
Family H/O & 32 & 16 & 14 & 7 & 0.311 \\
\hline
\end{tabular}

Kruskal Wallis test was used for numerical data. Post hoc was done in case of significant overall effect. Different letters indicate significant pairs. Categorical data was compared using Chi-square test.

Table (3) one -year outcome in patients of studied groups.

\begin{tabular}{llllll}
\hline & \multicolumn{2}{c}{ Total occlusion group } & \multicolumn{2}{c}{$\begin{array}{c}\text { Non-total occlusion } \\
\text { group }\end{array}$} & P value \\
\cline { 2 - 5 } & No & $\%$ & No & $\%$ & \\
\hline All cause of death & 14 & 7 & 12 & 6 & 0.847 \\
Cardiac death & 8 & 4 & 5 & 2.5 & 0.079 \\
Revascularization & 6 & 3 & 4 & 2 & 0.483 \\
Stroke & 21 & 10.5 & 25 & 12.5 & 0.114 \\
Stent thrombosis & 5 & 2.5 & 12 & 6 & 0.818 \\
Total MACE & 36 & 18 & 29 & 14.5 & 0.956 \\
\hline
\end{tabular}

Wang et al. [12] demonstrated that the offender conduit might have been blocked to $27 \%$ about NSTEMI patients Also that these patients needed bigger infarct span Furthermore higher six-month mortal sin.

In this study, we highlighted the contrasts in the benchmark aspects Furthermore clinical discoveries the middle of the to and non-TO gatherings. Patients with to were younger, were All the more often current smokers, Furthermore needed more level occurrence from claiming cardiovascular hazard factors, for example, hypertension Furthermore diabetes mellitus. These outcomes were over understanding with the individuals that bring been accounted Previously, registries contrasting STEMI What's more NSTEMI populaces [13-15]. Our discoveries might recommended that NSTEMI patients with on would often misdiagnosed What's more ought to be recognized as undetected Eventually Tom's perusing 12-lead ecg STEMI equivalents. However, it will be was troublesome will look at STEMI Furthermore NSTEMI patients who introduce for will due to those heterogeneities about these gatherings. Those whole NSTEMI populace comprises about different aggregations about patients. Those principle one assembly are patients for non-completely blocked IRAs and the individuals who best have subendocardial ischemia [3]. Another bunch may be created for patients for bit by bit expanding to Furthermore well-developed guarantees that keep transmural ischemia What's more ST-segment rise on ecg [16]. There would also NSTEMI patients for intense on of the infro- Also posterolateral circulations because of transmural ischemia that need aid not distinguished Eventually Tom's perusing ecg. It is probable that exactly NSTEMI patients for completely blocked offender courses supplying those posterolateral and subpar myocardia allotment the same 
pathophysiology with STEMI patients. This assembly might be misclassified because of the nonattendance about fitting precordial ecg heads that Might assistance recognizing will in this domain. The address stays if this misdiagnosis influences those conclusions. In the available analysis, Around NSTEMI patients for aggregate occlusion, $77.55 \%$ needed those offender confined in the infro- and posterolateral coursing library Also best 22 . $45 \%$ in the foremost coursing library. There were no contrasts in the short- alternately long haul mortal sin rates between these gatherings.

The average duration of the time from manifestations to angioplasty in the to bunch might have been $13.3 \mathrm{~h}$ and in the non-TO assembly might have been $15 \mathrm{~h}$. If those result might need been moved forward with prior mediation is was troublesome on assess. Current clinical rules clinched alongside NSTEMI don't propose schedule initial obtrusive strategy; the clinical trials didn't hint at any profit [1]. At there is an absence about information done patients for NSTEMI and should. Probably, on account for finish interference from claiming blood supply, fast rebuilding from claiming stream Might bring about more modest infarct span and superior prognosis. Our consider indicated that more than one-fourth of the NSTEMI number needed occlusive offender conduits. However, it will be was troublesome to foresee with of the ira for patients with NSTEMI. Previously, multivariate analysis, best those LCx Similarly as those offender lese greatness might have been distinguished Likewise a autonomous predictor from claiming on in the offender conduit. In the introduce contemplate those LCx constituted something like half from claiming instances for to in the NSTEMI assembly. It need been demonstrated that those vicinity from claiming ST fragment rise detects intense occlusions of the fellow Also RCA to $70 \%$ with $92 \%$ for cases, Yet just in $32 \%$ will $48 \%$ about instances to LCX-related myocardial localized necrosis [17-19]. More stupendous consideration about Doctors ought further bolstering a chance to be set On looking for STsegment sorrow clinched alongside heads V1-V3 Concerning illustration a marker LCx occlusion, particularly done patients for continuous indications [20].

Dependent upon time permits predictors and the set affectability from claiming surface ECG, it is was troublesome will distinguish intense aggregate coronary impediment clinched alongside NSTEMI patients. A standout amongst those supportive routines may be the utilization from claiming extra heads. Perron et al. [21] discovered that $\mathrm{s}$ were as from claiming seven altered prompts those standard 12 certain heads maximally builds those affectability of ecg to those identification of intense transmural ischemia for a negligible reduction from claiming specificity. It need likewise been accounted that a procedure known as figure surface possibility mapping enhances those identification about occlusions of the offender veins Previously, patients without ST-segment rise for starting 12-lead ECGs [22, 23]. Constitution surface possibility mapping Might expand STEMI identification by $27.5 \%$ In that acquired with standard ecg [24]. Moreover, transthoracic echocardiography may be helpful, especially to patients for non-evident transforms for ecg Furthermore separated parallel or posterior ischemia [25]. It need formerly been depicted that hypokinesis or akinesia of the ventricular myocardium gives the idea preceding the improvemen for ST-segment progressions on the ecg.

\section{Conclusion}

These patients in TO group had higher in-hospital outomes, but their long-term outcomes were similar to those of the non-TO group.

\section{References}

[1] C. W. Hamm, J. B. Bassand , S. Agewall ,ESC Committee for Practice Guidelines. ESC Guidelines for the management of acute coronary syndromes in patients presenting without persistent ST-segment elevation: The Task Force for the management of acute coronary syndromes (ACS) in patients presenting without persistent ST-segment elevation of the European Society of Cardiology (ESC). Eur Heart J, Vol. 32(23), PP. 2999-3054, 2011.

[2] P. H. G. Steg , S. K. James ,D. Atar , et al. Task Force on the management of ST-segment elevation acute myocardial infarction of the European Society of Cardiology (ESC). ESC Guidelines for the management of acute myocardial infarction in patients presenting with ST-segment elevation. Eur Heart J., Vol. 33(20), PP. 2569-2619, 2012.

[3] M. J. Davies, Pathophysiology of acute coronary syndromes. Indian Heart J,Vol. 52(4)PP. 473479 ,

[4] G. Wagner, T. Lim, Consideration of pitfalls in and omissions from the current ECG standards for diagnosis of myocardial ischemia/infarction in patients who have acute coronary syndromes. Cardiol Clin, gol, Vol. 24(3), PP. 331-42, .

[5] L. Poloński ,M. Gasior ,M. Gierlotka , Polish Registry of Acute Coronary Syndromes (PLACS). Characteristics, treatments and outcomes of patients with acute coronary syndromes in Poland. Kardiovol Vol. Po65(8): 861-873, indexed in Pubmed: 17853315.

[6] Flynn MR, Barrett C, Cosío FG, et al. The Cardiology Audit and Registration Data Standards (CARDS), European data standards for clinical cardiology practice. Eur Heart, Vol. 26(3), PP. 308-313,2015 .

[7] C. Baigent, R.Collins ,p. Appleby, ISIS-2: 10 year survival among patients with suspected acute myocardial infarction in randomised comparison of intravenous streptokinase, oral aspirin, both, or neither. The ISIS-2 (Second International Study of Infarct Survival) Collaborative Group, Vol. 316(7141), PP.1337-134310, 2012.

[8] M. G. Franzosi, E. Santoro,C. De Vita, Ten-year follow-up of the first megatrial testing thrombolytic therapy in patients with acute 
myocardial infarction: results of the Gruppo Italiano per lo Studio della Sopravvivenza nell'Infarto-1 study. The GISSI Investigators. Circulatio,Vol. 98(24)PP. 2659-2665.,2015,

[9] J. W. Hurst, Thoughts about the abnormalities in the electrocardiogram of patients with acute myocardial infarction with emphasis on a more accurate method of interpreting ST-segment displacement: part I. Clin Cardiol, Vol. 30(8), PP. 381-390, 2007,

[10] A. Krishnaswamy, A. M. Lincoff, Magnitude and consequences of missing the acute infarct-related circumflex artery. Am Heart, Vol. 158(5), PP. 706-712, 2006, .

[11] P. Bahrmann,J. Rach,S. ppDesch, Incidence and distribution of occluded culprit arteries and impact of coronary collaterals on outcome in patients with non-ST-segment elevation myocardial infarction and early invasive treatment strategy. Clin Res Cardiol ,vol100(5), PP. 457467,2017.

[12] T. Y. Wang, M. Zhang , Y. Fu, . Incidence, distribution, and prognostic impact of occluded culprit arteries among patients with non-STelevation acute coronary syndromes undergoing diagnostic angiography. Am Heart J157(4), PP. 716-723, 2009.

[13] J. D. Abbott,H. N. Ahmed ,H. A.Vlachos , Comparison of outcome in patients with STelevation versus non-ST-elevation acute myocardial infarction treated with percutaneous coronary intervention (from the National Heart, Lung, and Blood Institute Dynamic Registry). Am J Cardiol, Vol.100(2), PP. 190-195, 2017

[14] L. Polonski , M. Gasior ,M.Gierlotka ,PL-ACS Registry Pilot Group. A comparison of ST elevation versus non-ST elevation myocardial infarction outcomes in a large registry database: are non-ST myocardial infarctions associated with worse long-term prognoses? Int J Cardiol,Vol.152(1), PP. 70-77.

[15] R. J. Goldberg, K. Currie , S. White, Six-month outcomes in a multinational registry of patients hospitalized with an acute coronary syndrome (the Global Registry of Acute Coronary Events [GRACE]). Am J Cardiol,Vol. 93(3), PP. 288293, 2004.

[16] E. M. Antman, E. Braunwald, Acute myocardial infarction. In: Braunwald E, Zipes DP, Libby P, eds. Heart disease: A Textbook of Cardiovascular Medicine. 6th Ed. Philadelphia, PA: WB Saunders Co Ltd, Vol. 22, PP. 1114-1251, 2012.
[17] B. L. Huey, G. A. Beller, KDL, A comprehensive analysis of myocardial infarction due to left circumflex artery occlusion: comparison with infarction due to right coronary artery and left anterior descending artery occlusion. J Am Coll Cardio, Vol. 12(5), PP. 1156-1166, 2015.

[18] C. Schmitt ,K. Lehmann , S. Schmieder, Diagnosis of acute myocardial infarction in angiographically Documented Occluded Infarct Vessel. Chest, Vol. 120, PP. 1540-15462001, 1990.

[19] C. Berry , a. Zalewski , r. Kovach , Surface electrocardiogram in the detection of transmural myocardial ischemia during coronary artery occlusion. Am J Cardiol, Vol. 363(1), PP. 21, 2003.

[20] K. Thygesen ,a. Jaffe, Writing Group on the Joint ESC/ACCF/AHA/WHF Task Force for the Universal Definition of Myocardial Infarction, ESC Committee for Practice Guidelines (CPG). Third universal definition of myocardial infarction. Eur Heart J, Vol. 33(20), PP. 25512567,2012.

[21] A. Perron, T. Lim , u. Pahlm-Webb, Maximal increase in sensitivity with minimal loss of specificity for diagnosis of acute coronary occlusion achieved by sequentially adding leads from the 24-lead electrocardiogram to the orderly sequenced 12-lead electrocardiogram. J Electrocardiol,Vol. 40(6), PP. 463-469,2007.

[22] M. J. Daly, D. D. Finlay ,D. Guldenring , Detection of acute coronary occlusion in patients with acute coronary syndromes presenting with isolated ST-segment depression. Eur Heart J Acute Cardiovasc Care, Vol. 1(2), PP. 128-135, 2007.

[23] G. Owens, A. McClelland, S. Walsh, Comparison of value of leads from body surface maps to 12lead electrocardiogram for diagnosis of acute myocardial infarction. Am J Cardiol, Vol. 102(3): PP. 257-265, 2008.

[24] J. W. Hoekstra, B. J. O’Neill , Y. B. Pride, Acute detection of ST-elevation myocardial infarction missed on standard 12-Lead ECG with a novel 80-lead real-time digital body surface map: primary results from the multicenter OCCULT MI trial. Ann Emerg Med, Vol. 54(6), PP. 779788.e1,2009.

[25] B. C. Corya Applications of echocardiography in acute myocardial infarction. Cardiovasc Clin, Vol. 7(2), PP. 113127,1975. 